Eur J Health Econom 2005 · 50:244-252 DOI 10.1007/s10198-005-0297-y

Published online: 4. May 2005

(c) Springer Medizin Verlag 2005

\author{
Beate Sander 1,5 - Marlene Gyldmark ${ }^{2}$. Frederick G. Hayden ${ }^{3} \cdot$ James Morris $^{4}$ \\ Elvira Mueller ${ }^{1} \cdot$ Rito Bergemann ${ }^{1}$ \\ ${ }^{1}$ Institute for Medical Outcome Research GmbH, Lörrach, Germany \\ ${ }^{2}$ Health Economics and Strategic Pricing, F. Hoffmann-La Roche Ltd Pharmaceuticals \\ Division, Basel, Switzerland \\ ${ }^{3}$ Division of Infectious Diseases and International Health, School of Medicine, \\ University of Virginia, Charlottesville, VA, USA \\ ${ }^{4}$ Roche Products Ltd, Welwyn, UK \\ ${ }^{5}$ Division of Clinical Decision-Making \& Health Care Research, \\ University Health Network, Toronto, ON, Canada
}

\title{
Influenza treatment with neuraminidase inhibitors
}

\section{Cost-effectiveness and cost-utility in healthy adults in the United Kingdom}

nfluenza is well known among the general public with regard to its epidemic occurrence and association with disabling respiratory illness. However, people are considerably less aware of the size and distribution of the economic burden imposed by this virus. A recent study of the medical care use related to influenza in the United Kingdom during the mid-1990s found that in absolute terms adults aged 15-64 years had more influenza-related physician visits and clinical complications and greater drug use than children or the elderly [1].

This study explored the cost-effectiveness of treating an otherwise healthy adult population for influenza during a typical annual epidemic period. The treatment interventions investigated are oseltamivir and zanamivir, neuraminidase inhibitors that work specifically against influenza type $A$ and $B$ by inhibiting the replication of the virus in the respiratory tract. Both are administered twice daily for 5 days. Oseltamivir is taken orally, whereas zanamivir is inhaled using a special device. The treatment needs to be initiated within $48 \mathrm{~h}$ of onset of symptoms for efficacy and usually is associated with only mild, transient side effects if used as prescribed. Two other competing products exist in some mar- kets: amantadine and rimantadine (not in the UK), both so-called M2 ion channel blockers that only work against influenza A and are associated with gastrointestinal and central nervous system side effects. Amantadine is not recommended by the National Institute for Clinical Excellence (NICE) for influenza treatment [2]. Given that most influenza cases are treated with over-the-counter (OTC) medication only, we chose to compare usual care with oseltamivir and zanamivir.

\section{Methods}

\section{The decision-analytic model}

A pharmacoeconomic decision-analytic model incorporating first- and secondorder Monte Carlo simulation was developed to investigate cost-effectiveness and cost-utility for a healthy adult population (13-64 years) both from the perspective of the healthcare payer [UK National Health Service (NHS)] and from the societal perspective (considers all costs and health benefits regardless of who experiences them). For the purposes of this analysis usual care, defined as symptomatic treatment, is the baseline strategy, since this remains standard practice in influenza treatment despite the availability of alternatives.

The decision tree (• Fig. 1) visualizes morbidity and mortality due to influenza and its specified complications. The model starts at the general practitioner's (GP) office since both oseltamivir and zanamivir are available only with a GP's prescription and not as OTC medication. The treatment options are usual care (current practice), oseltamivir (intervention 1) and zanamivir (intervention 2). All effects of intervention with oseltamivir or zanamivir are those achieved in addition to the use of OTC medication, as use of selected OTC medicines (paracetamol, antitussives) was allowed in the clinical trials for disabling symptoms.

The model distinguishes between two periods for the start of treatment (within $48 \mathrm{~h}$ of onset of symptoms and after $48 \mathrm{~h}$ ) as well as between influenza-positive and influenza-negative cases (diagnostic certainty) since treatment with neuraminidase inhibitors is confirmed to be effective against influenza virus only if taken within $48 \mathrm{~h}$ of onset of symptoms. Costs of oseltamivir or zanamivir treatment are incurred although there is no improvement in health outcome. Each infected individ- 


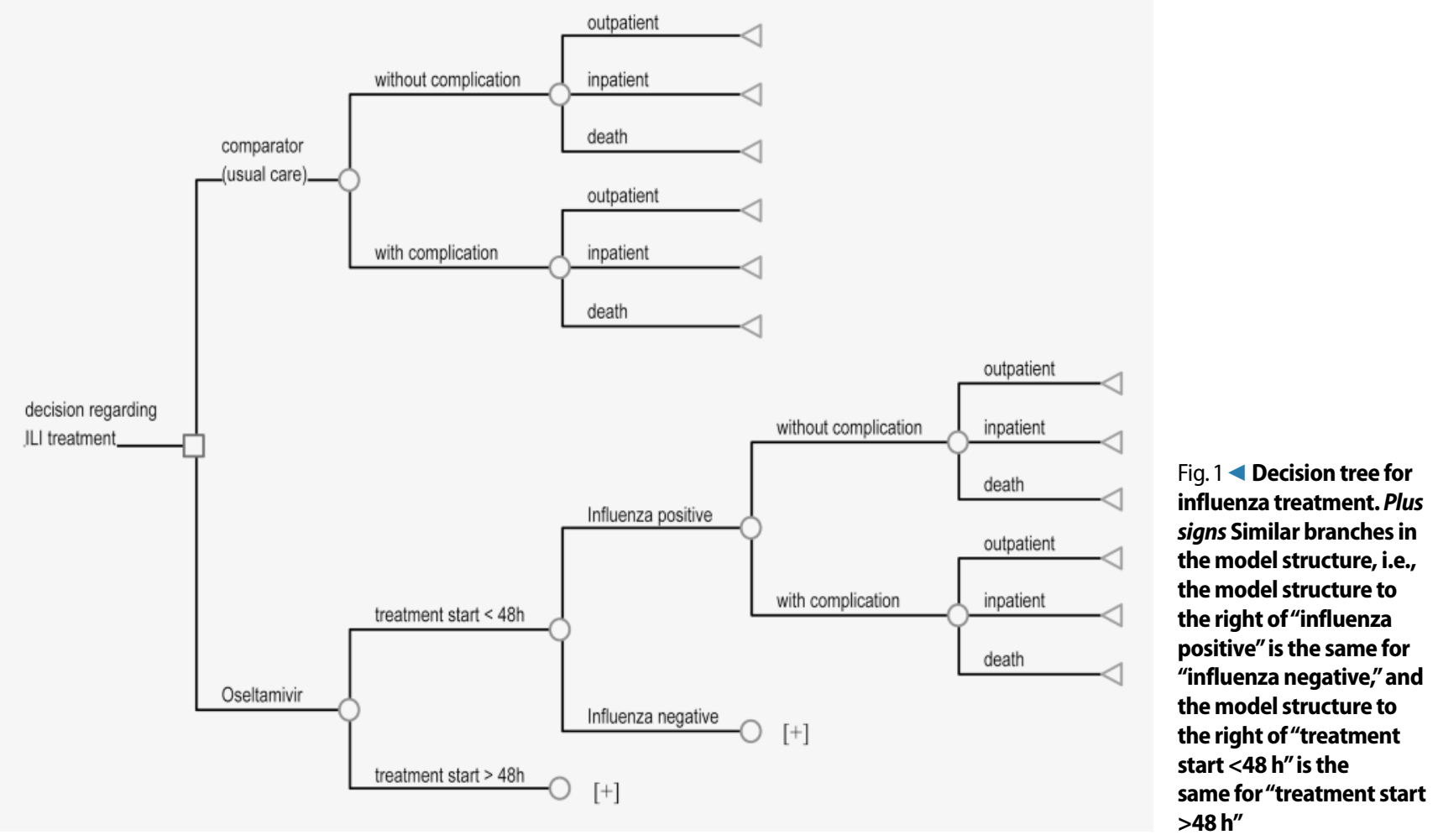

ual has probabilities of having influenzalike illness (ILI) only or of developing ILIrelated complications such as pneumonia and bronchitis.

For both patients with and those without complications the model then describes three disease states: outpatient (symptomatic infection that results in outpatient treatment in the primary sector), inpatient (symptomatic infection that results in hospitalization, including outpatient treatment pre- and posthospitalization), and death (symptomatic infection that results in death). Only deaths related to ILI or to its complications are included in the model (including outpatient and inpatient treatment before death). The same disease states apply for all ILI cases (i.e., influenza virus positive as well as influenza virus negative cases). Similar branches are represented by a plus sign in the model structure.

\section{Data input}

The model is based on published epidemiological and clinical trial data $[1,3,4,5,6$, $7,8,9,10,11,12,13,14,15,16,17,18]$. A Medline search was performed covering a variety of epidemiological/medical as well as health economic keywords for the years
1997-2003. After applying inclusion and exclusion criteria such as quality of study, outcome parameters measured, and comparability across studies, only a small fraction of the retrieved literature was used as a final data source for the model. Clinical trial data and literature data were complemented with assumptions, supported by expert opinion. Generally, epidemiological data were used for usual care and the relative risk reduction, and relative improvement were applied to estimate events for oseltamivir- and zanamivir-treated patients. Country-specific resource use and cost data were collected separately from published sources $[9,10,19]$.

\section{Probabilities}

- Table 1 presents the main probabilities used in the analysis. The two complications included in this analysis - bronchitis and pneumonia - were chosen because of their strong association with influenza, their high incidence, and their applicability to the chosen population group [20]. The model assumes that individuals can develop only a single complication. The underlying data from epidemiological studies provide the incidence of each complication. There is no published information on whether some individuals in the study population had more than one complication at a time. It is assumed that each reported complication occurred in a different patient. Treatment with neuraminidase inhibitors is assumed to have an effect on the incidence of complications but not on the course of these. This means baseline (usual care) and interventions (neuraminidase inhibitors) have the same probabilities for hospitalization and mortality due to bronchitis or pneumonia once these complications develop. Due to limited data availability further assumptions have been made: no hospitalization is assumed for bronchitis and the mortality rate due to a complication is not lower than that of influenza in itself. The impact of the uncertainty surrounding the data used for these variables (probability of complication, hospitalization, mortality) is determined in probabilistic sensitivity analysis (secondorder Monte Carlo simulation).

There is recent evidence that in clinical practice compliance with zanamivir is an issue, particularly in elderly patients, where the majority (65\%) has been found to have difficulty loading the device [21]. However, clinical trials in healthy adult populations have reported high rates of compliance, and therefore $100 \%$ compliance for zanamivir is assumed. The possi- 
Eur J Health Econom $2005 \cdot 50: 244-252$

DOI 10.1007/s10198-005-0297-y

๑) Springer Medizin Verlag 2005

Beate Sander · Marlene Gyldmark

Frederick G. Hayden · James Morris

Elvira Mueller $\cdot$ Rito Bergemann

\section{Influenza treatment with neuraminidase inhibitors. Cost-effectiveness and cost-utility in healthy adults in the United Kingdom}

\begin{abstract}
We assessed the cost-effectiveness and costutility of treating influenza with neuraminidase inhibitors (oseltamivir and zanamivir) from a health care payer's and societal perspective in the United Kingdom. A simulation model was developed to predict morbidity and mortality due to influenza and its specified complications, comparing neuraminidase inhibitors with usual care in an otherwise healthy adult population. Robustness of the results was tested by one-way and multiway as well as probabilistic sensitivity analyses. Treatment with either neuraminidase inhibitor results in reduced morbidity and faster return to normal activities. However, oseltamivir dominates zanamivir in cost-utility analysis due to its lower costs. Comparing oseltamivir with usual care, the costs are $£ 14.36$ per day of normal activity gained and $£ 5,600$ per quality-adjusted lifeyear gained from the healthcare payer perspective. Oseltamivir dominates usual care from the societal perspective. Treatment with oseltamivir is a cost-effective strategy for otherwise healthy adults in the UK from both the healthcare payer and societal perspective.
\end{abstract}

\section{Keywords}

Influenza - Treatment - Antivirals .

Cost-effectiveness · Decision modeling bility that some patients discontinue treatment when they feel better, or if they develop gastrointestinal side effects under oseltamivir treatment is already included in the underlying clinical trial data as the intend to treat population was used.

Drug side effects are usually mild and self-limiting i.e., there is no resource use associated with them. The impact of side effects on quality of life is already included in the daily quality of life reported in the clinical trials.

\section{Costs and resource use}

This study includes an assessment of direct medical costs (healthcare payer perspective) but also of productivity loss due to sick leave based on unit costs from the year 2001 (- Table 2). Direct healthcare costs used in the model include drug use costs and primary and secondary care resource costs. A comprehensive United States database [National Ambulatory Medical Care Survey (NAMCS)] has been used to develop estimates of the resource use of each complication, including GP and specialist visits, tests and investigations, and drugs [10]. Data to this level of detail were not available specifically for the UK. The NAMCS data provide rates of drug prescribing by complication as well as drug class. Overall rates of antibiotic prescribing in the NAMCS database were strikingly similar to published estimates in the UK. For example, Meier et al. [1] reported that approx. $80 \%$ of complicated influenza cases in the UK are treated with an antibiotic; the NAMCS estimate was $83 \%$. For uncomplicated influenza cases the rates were $40 \%$ and $36 \%$, respectively. Productivity loss was valued by applying the human capital approach thus using the time to return to normal activity (days) as evaluated in epidemiological studies and clinical trials and valuing the time with the average gross UK income.

\section{Health outcome: days of normal activity gained}

Time to return to normal activity was chosen as the effectiveness measure since it is the measure that is most meaningful to the patient as well as to employers and decision makers. Alleviation of fever or symptoms captures only part of the disease picture, with return to normal activity being the measure that best captures the "cure" of the disease and the total duration of illness.

Time to return to normal activity was chosen over mortality as a measure of effectiveness, as mortality is very low in healthy adults and therefore is not an endpoint in clinical trials in the healthy adult population. The main goal in treating healthy adults is to restore the patients' health as fast as possible. Furthermore, mortality is included in the quality adjusted life years.

Time to return to normal activity was a secondary clinical endpoint in the oseltamivir trials and was measured using an 11-point Likert-type scale in which the endpoint 10 was "returned to normal activity." Normal activity was defined as usual ability to carry out normal activities; this is of course dependent on the individual and his/her own interpretation. In the healthy adults trials most patients returned to normal activity within the 21 day followup period (after treatment start); however, the means are restricted means as a few patients had not returned to normal activities before last trial measurement. This means the patients not returning to normal activity within 21 days were censored. The zanamivir trial, however reported the median duration of days to return to normal activity: 8.5 days (placebo) vs. 7 days (zanamivir), a reduction of $18 \%$. Patients recorded their symptoms, temperature, and ability to perform normal daily activities on a diary card for 14 days, if symptoms persisted for 28 days [17].

\section{Health outcome: quality-adjusted life-years saved}

Both avoided death and improved quality of life can be given a single value using the approach of quality-adjusted life-years (QALYs). A quality weight is assigned to each health state and adjusted for the time a patient spends in this state. This quality weight is a value between o (death) and 1 (perfect health).

Quality weights were derived from the literature for all diseases included in the analysis (influenza, bronchitis, pneumonia). The same source was used to ensure consistency between the weights [22]. These are generically derived quality weights, which means they are also consistent with quality weights of various other diseases such as heart disease or cancer. 
Table 1

Model inputs: probabilities (IL/ influenzalike illness)

\begin{tabular}{|c|c|c|c|c|}
\hline Variable & Treatment & $\begin{array}{l}\text { Probability } \\
\text { (mean) }\end{array}$ & Distribution & Reference \\
\hline \multirow{3}{*}{ ILI inpatient } & - Usual care & 0.00323 & Beta $(0.00323 ; 0.99677$ & [4] \\
\hline & - Oseltamivir & 0.00116 & Beta $(0.00116 ; 0.99884)$ & $64 \%$ reduction vs. usual care [14] \\
\hline & -Zanamivir & 0.00116 & Beta $(0.00116 ; 0.99884)$ & $\begin{array}{l}\text { Assumed to have same reduction as oseltamivir, although } \\
\text { no difference to placebo detected in clinical trials [5] }\end{array}$ \\
\hline \multirow[t]{3}{*}{ ILI death } & - Usual care & 0.00002 & Beta $(0.00002 ; 0.99998)$ & [18] \\
\hline & - Oseltamivir & 0.00002 & Beta $(0.00002 ; 0.99998)$ & Assumption: no reduction in ILI-related mortality \\
\hline & -Zanamivir & 0.00002 & Beta $(0.00002 ; 0.99998)$ & Assumption: no reduction in ILI-related mortality \\
\hline \multirow[t]{3}{*}{ Bronchitis } & - Usual care & 0.01234 & Beta $(0.01234 ; 0.98766)$ & [1] \\
\hline & - Oseltamivir & 0.00802 & Beta $(0.00802 ; 0.99198)$ & $35 \%$ reduction vs. usual care [14] \\
\hline & -Zanamivir & 0.00759 & Beta $(0.00759 ; 0.99241)$ & $39 \%$ reduction vs. usual care (all adults) [5] \\
\hline Bronchitis inpatient & All & 0.00323 & Beta $(0.00323 ; 0.99677$ & Assumption: influenza \\
\hline Bronchitis death & All & 0.00005 & Beta $(0.00005 ; 0.99995)$ & Assumption: influenza \\
\hline \multirow[t]{3}{*}{ Pneumonia } & -Usual care & 0.00278 & Beta $(0.00278 ; 0.99722)$ & [1] \\
\hline & - Oseltamivir & 0.00067 & Beta $(0.00067 ; 0.99933)$ & $76 \%$ reduction vs. usual care [14] \\
\hline & -Zanamivir & 0.00132 & Beta $(0.00132 ; 0.99868)$ & $53 \%$ reduction vs. usual care (all adults) [5] \\
\hline Pneumonia inpatient & All & 0.07293 & Beta $(0.07293 ; 0.92707)$ & [6] \\
\hline Pneumonia death & All & 0.01883 & Beta $(0.01883 ; 0.98167)$ & [7] \\
\hline \multirow{3}{*}{$\begin{array}{l}\text { Days to return to nor- } \\
\text { mal activity }\end{array}$} & - Usual care & 8.83 & Gamma $(6.63 ; 11.04)$ & Roche clinical trials \\
\hline & - Oseltamivir & 7.43 & Gamma $(5.57 ; 9.29)$ & $16 \%$ reduction vs. usual care (Roche clinical trials) \\
\hline & -Zanamivir & 7.27 & Gamma $(5.85 ; 9.74)$ & $18 \%$ reduction vs. usual care [17] \\
\hline Quality weights & Oseltamivir & 7.43 & - & - \\
\hline \multirow[t]{3}{*}{ ILI } & -Zanamivir & 7.79 & None & {$[22]$} \\
\hline & - Oseltamivir & 0.94 & None & $\begin{array}{l}12 \% \text { improvement vs. usual care over the first } 9 \text { days, } \\
\text { after } 9 \text { days no further benefit assumed (Roche Clinical } \\
\text { trials, similar to [23]) }\end{array}$ \\
\hline & -Zanamivir & 0.94 & None & $\begin{array}{l}\text { Assumed to have same improvement as oseltamivir as } \\
\text { quality of life has not been evaluated in zanamivir clinical } \\
\text { trials }\end{array}$ \\
\hline Bronchitis & All & 0.99 & None & [22] \\
\hline Pneumonia & All & 0.90 & None & {$[22]$} \\
\hline
\end{tabular}

Since the quality of life varies between the different treatment options for influen$\mathrm{za}$, the results of clinical trials (Roche [15]) were used to estimate the relative improvement in quality of life between placebo and oseltamivir during the first 7 days of the influenza episode. The same approach as in a recent NICE assessment [23] was used: the improvement in quality of life between placebo and oseltamivir was estimated from patient health state valuations reported daily for 21 days in oseltamivir clinical trials. QALYs were generated by recalibrating the Roche Likert score to mean vsual analogue scale (VAS) scores and then transforming them to time trade-off equivalent scores (for a description of the transformation see [15]). The relative im- provement for an otherwise healthy adult population was applied to the generic influenza quality weight [22] to derive a quality weight for ILI for patients effectively treated with oseltamivir. As no data are available on quality of life improvement for zanamivir, the same improvement as for oseltamivir was assumed.

\section{Modeling approach}

\section{First-order Monte Carlo simulation}

Simulated patients are followed through the model individually, and since an individual patient can be only in a single state at any given time, he can pass down only one branch of the tree at a given chance node (• Fig. 1). Hence the path followed by different patients differs due to chance. A large number of patients $(10,000)$ was chosen because of the large number of endpoints and the very small probabilities in the model, especially for mortality. The individual simulation method estimates the likely variance that is related simply to the inherent uncertainty of the probabilistic structure of the model (patient variability) [24].

\section{Second-order Monte Carlo simulation}

Second-order Monte-Carlo simulation models deal with the uncertainty related to input variables using probability distributions around their point values. This means that in addition to allowing for vari- 
Table 2

Model inputs: unit costs, based on the year 2001 (ILI influenzalike illness,

OTC over-the-counter)

\begin{tabular}{|c|c|c|c|}
\hline Variable & Unit cost ( $($ ) & Unit & References \\
\hline Oseltamivir & 18.18 & Episode & Roche UK \\
\hline \multirow[t]{2}{*}{ Zanamivir } & $\cdot 24.00$ & Episode & [8] \\
\hline & - 6.50 & Episode & $\begin{array}{l}15 \text { min nursing time for instruction } \\
\text { on device use (expert opinion) }\end{array}$ \\
\hline General practitioner visit & 19.00 & Visit & [9] \\
\hline Specialist visit & 74.00 & Visit & [9] \\
\hline \multicolumn{4}{|l|}{ Tests } \\
\hline - Bronchitis & 28.48 & Episode & [10] \\
\hline - Pneumonia & 38.00 & Episode & [10] \\
\hline \multicolumn{4}{|l|}{ Antibiotics } \\
\hline •ILI & 6.60 & Episode & [8] \\
\hline - Bronchitis & 6.60 & Episode & {$[8]$} \\
\hline - Pneumonia & 23.40 & Episode & [8] \\
\hline OTC & 5.00 & Episode & Assumption \\
\hline Hospitalization & 242.00 & Day & [9] \\
\hline Hourly wage & 10.53 & Hour & [11] \\
\hline
\end{tabular}

ability due to the way in which individuals travel through the model (first-order Monte Carlo simulation) the underlying model variables are allowed to vary over a given range with a given distribution. For each simulation run a value from the distribution of each variable is picked at random, generating one set of outputs (costs and effectiveness). A large number of simulation runs therefore yields a distribution of outputs [25]. Because of their possible impact and the uncertainty surrounding them certain variables were chosen from predefined distributions: probabilities (complication, hospitalization, mortality) and duration (days to return to normal activity, length of stay in hospital). Beta distributions (probabilities) and gamma distributions (duration) were used. Each of the 10,00o patients randomly traverses a single path through the model. The model is recalculated 100 times. Incremental costeffectiveness and cost-utility analyses are provided of additional costs and additional health outcomes associated with the scenarios compared.

Two perspectives are investigated: healthcare payer and society. The human capital approach, based on average income, is used to value a patient's inability to work due to illness $[26,27]$. To avoid double counting in the cost-utility analysis, indi- rect costs due to premature mortality are not included since these are captured by the measure of health outcomes (QALYs) [28]. For comparison these costs were also excluded in the cost-effectiveness analysis. Most costs and effects related to influenza occur within 1 year but some occur in the future (years of life lost due to premature death). Therefore results of this analysis are presented as discounted using a discount rate of $1.5 \%$ for health outcomes (years of life lost) per annum, which is recommended for economic evaluations in the healthcare field in the UK [29].

\section{Analyses}

The base case is defined as having a $70 \%$ diagnostic certainty rate and $100 \%$ treatment start within $48 \mathrm{~h}$ from onset of symptoms. One-way sensitivity analyses are presented for two variables that are generally believed to have a major impact on the results: diagnostic certainty and time of treatment start after onset of symptoms. Diagnostic certainty describes the percentage of influenza-positive patients within the ILI population, which is presumably higher when influenza is circulating. For the base case scenario diagnostic certainty is assumed to be $70 \%$ (point estimate). This assumption was made based on a diagnos- tic certainty rate of around $67 \%$ observed in oseltamivir clinical trials [30]. Drawing on analyses published for the zanamivir technology assessment, $34 \%$ was chosen as a low estimate of diagnostic certainty in sensitivity analysis [19]. Since time of treatment start should be within $48 \mathrm{~h}$ of onset of symptoms for treatment with neuraminidase inhibitors to be effective, the assumption is made that only patients having a GP consultation within this period receive the neuraminidase inhibitors. In sensitivity analysis the impact of a proportion of patients receiving the neuraminidase inhibitors after $48 \mathrm{~h}$ of symptoms was evaluated. Clinical trials are likely to have controlled for this factor already to some extent. However, the impact of $25 \%$ of patients receiving treatment in excess of $48 \mathrm{~h}$ after symptom onset was tested. This represents an arbitrary proportion of late presenters receiving treatment, but helps to demonstrate the sensitivity of results to this variable.

Multiway sensitivity analysis, assuming neuraminidase inhibitors have no effect on hospitalizations, complications, or mortality and a low diagnostic certainty rate of $34 \%$, was performed as these outcome measures have not been included to varying degrees in other publications. This means the only effect captured is the reduction in days to return to normal activity and the improvement in quality of life of a typical outpatient episode of influenza. An additional sensitivity analysis shows results excluding all hospitalizations, complications, and mortality from all strategies (usual care, oseltamivir and zanamivir) with a diagnostic certainty rate of $34 \%$.

Only life years lost due to premature death are discounted. The discount rate is therefore expected to have only a small impact on the results. However, an undiscounted analysis was performed. Days lost (time to return to normal activity) are valued using the human capital approach. While this approach reflects value of healthy time foregone, i.e., also the value of missing leisure time, time to return to normal activity as used in the base case analysis seems long compared to that in other studies evaluating productivity loss [31]. Sensitivity analysis was therefore performed valuing only productivity loss by assuming that for outpatients only 
$25 \%$ of the time to return to normal activities is productivity loss and that for inpatients weekends would usually (i.e., if not sick) be leisure time and are therefore no work loss.

\section{Results}

- Table 3 presents the expected costs and benefits in the base case scenario for the treatment of otherwise healthy adults. From the healthcare payer perspective oseltamivir is associated with greater overall benefits than zanamivir and usual care, greater mean expected cost per patient than usual care, and lower mean expected cost per patient than zanamivir. Differences in costs between zanamivir and oseltamivir are due mainly to the higher price of zanamivir but also to a higher rate of pneumonia (zanamivir). Oseltamivir therefore dominates zanamivir in cost utility analysis. The incremental cost per day of normal activity gained with oseltamivir in comparison to usual care is $£ 14.36$ (minimum: $£ 10.69$, maximum: $£ 17.67)$ and the mean expected incremental cost per discounted QALY is $\mathfrak{E 5}_{5}, 600$ (minimum: $£ 1,403$, maximum: usual care dominant).

- Figure 2 presents a cost-effectiveness acceptability curve for the comparison oseltamivir to usual care (health care payer perspective) generated by probabilistic analyses, showing the level of uncertainty around the base case estimate of cost-effectiveness. The probability of oseltamivir being cost-effective in the otherwise healthy adult population at an assumed cost per QALY ceiling of $£ 30$,000 is 0.85 . Analyses were also conducted from the societal perspective (• Table 3). Oseltamivir dominates usual care but also zanamivir in costutility analysis, accruing more benefits per patient at less cost.

\section{Sensitivity analyses}

Results of one-way and multiway sensitivity analyses are presented for the comparison oseltamivir to usual care only since zanamivir is dominated by oseltamivir in sensitivity analyses because zanamivir is more expensive than oseltamivir in the UK, while the two neuraminidase inhibitors have a similar way of action and effectiveness. The impact of a lower level of

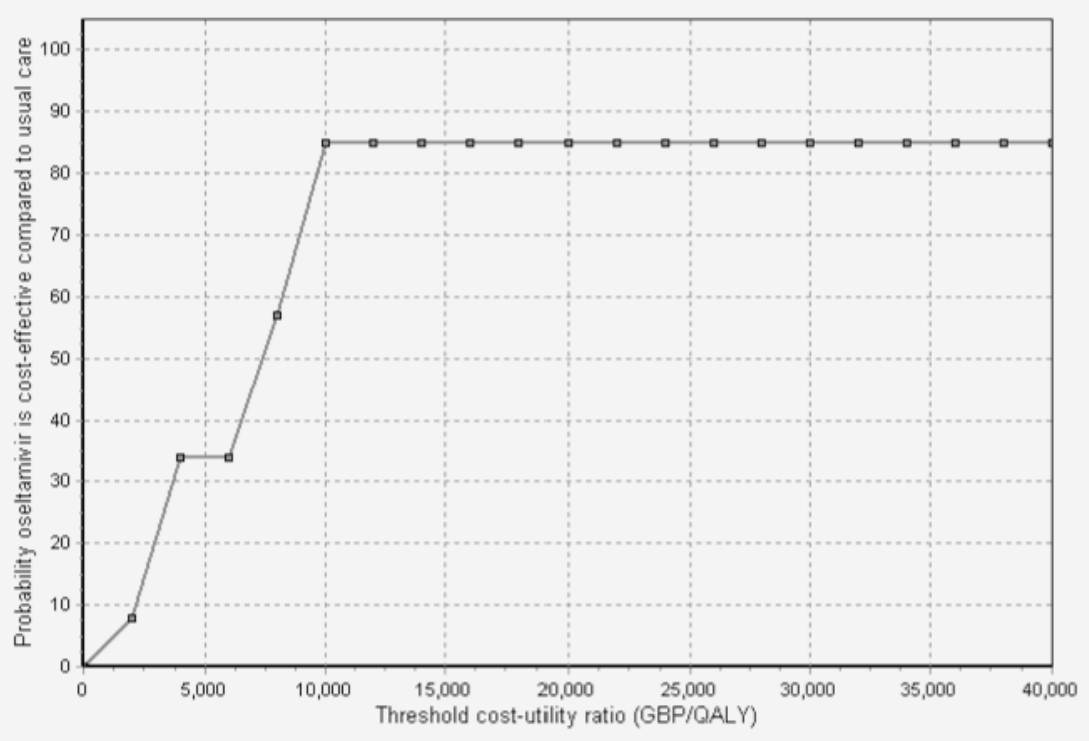

Fig. $2 \Delta$ Cost-acceptability curve for the health care payer perspective. Base case scenario; comparison oseltamivir to usual care. GBP Pounds sterling

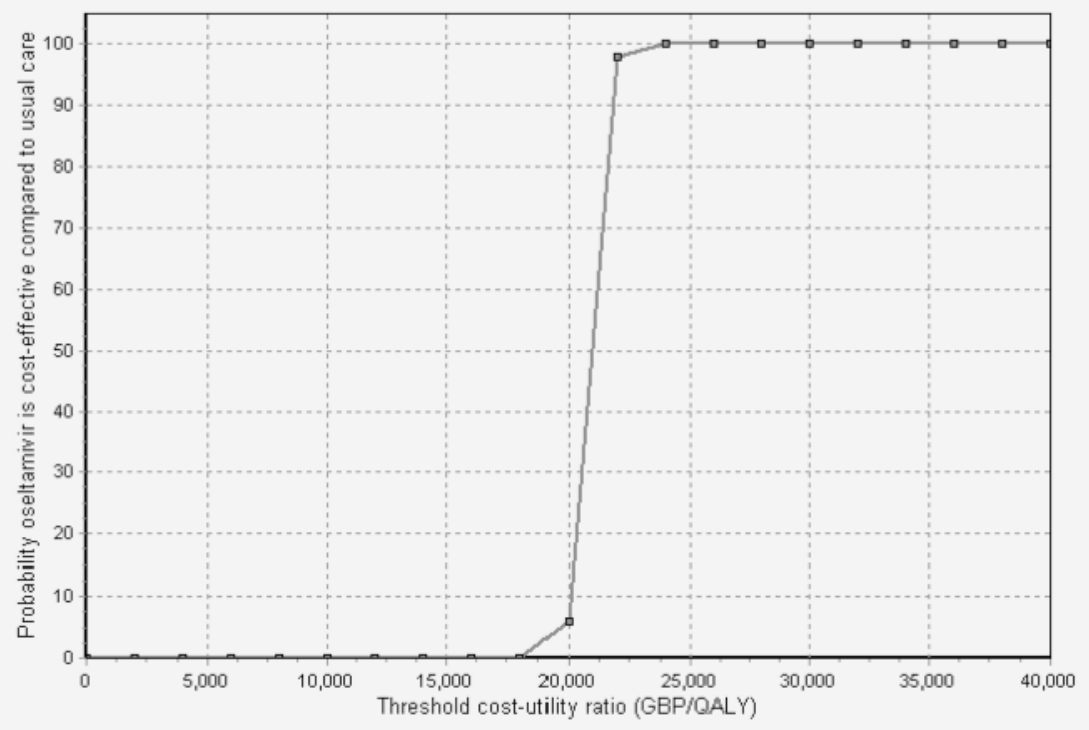

Fig. $3 \Delta$ Cost-acceptability curve for the health care payer perspective. Sensitivity analysis: neuraminidase inhibitors have no effect on hospitalizations, complications, mortality, diagnostic certainty $34 \%$; comparison oseltamivir to usual care. GBP Pounds sterling

diagnostic certainty upon the incremental cost-effectiveness of oseltamivir was compared to usual care (see $\bullet$ Table 4). For the NHS perspective the analyses demonstrate that the results are very sensitive to the level of diagnostic certainty assumed. As the level of diagnostic certainty is reduced, the incremental cost-effectiveness ratios for oseltamivir increase. This can also be seen in a shift of the cost-acceptability curve to the right (see - Fig. 3). However, oseltamivir remains cost-effec- tive in terms of the incremental cost per QALY gained from the healthcare payer's perspective at a threshold of 30,000/QALY gained and dominant from the societal perspective even assuming 34\% diagnostic certainty.

The impact of time to treatment initiation on cost-effectiveness results was also assessed (- Table 4). In the base case we assume that $100 \%$ of patients treated with oseltamivir start their treatment within $48 \mathrm{~h}$ of symptom onset. For the sensitivity 
Table 3

Base case incremental cost-effectiveness results: means (parentheses minimum and maximum) (D dominates in the incremental analysis, UC usual care, Oselt. oseltamivir)

\begin{tabular}{|c|c|c|c|c|c|}
\hline & Cost per patient ( $(£)$ & $\begin{array}{l}\text { Time to return } \\
\text { to normal } \\
\text { activity per } \\
\text { patient (days) }\end{array}$ & $\begin{array}{l}\text { Cost per day of normal } \\
\text { activity gained ( }(£)\end{array}$ & QALYs (discounted) & 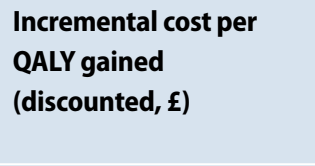 \\
\hline \multicolumn{6}{|c|}{ Health care payer perspective } \\
\hline - Usual care & $50.66(47.73 ; 54.53)$ & $8.96(8.93 ; 8.99)$ & - & $31.3797(31.3726 ; 31.3818)$ & - \\
\hline - Oseltamivir & $65.24(63.67 ; 66.83)$ & $7.95(7.92 ; 7.98)$ & $14.36(10.69 ; 17.67)$ & $31.3823(31.3714 ; 31.3836)$ & $5,600(1,403 ;$ UC D) \\
\hline -Zanamivir & $77.54(75.88 ; 79.81)$ & $7.84(7.81 ; 7.87)$ & $112.84(93.88 ; 149.67)$ & $31.3821(31.3745 ; 31.3836)$ & Oselt. D (3,737; Oselt. D) \\
\hline \multicolumn{6}{|c|}{ Society perspective } \\
\hline - Usual care & $808.23(803.97 ; 813.30)$ & $8.96(8.93 ; 8.99)$ & - & $31.3797(31.3726 ; 31.3818)$ & - \\
\hline - Oseltamivir & $736.96(733.87 ; 740.57)$ & $7.95(7.92 ; 7.98)$ & Oselt. D (Oselt. D; Oselt. D) & $31.3823(31.3714 ; 31.3836)$ & Oselt. D (Oselt. D; 15,619 ${ }^{b}$ ) \\
\hline -Zanamivir & $740.03(736.20 ; 743.60)$ & $7.84(7.81 ; 7.87)$ & $28.19(8.00 ; 56.75)$ & $31.3821(31.3745 ; 31.3836)$ & Oselt. D (318.54; Oselt. D) \\
\hline
\end{tabular}

Table 4

Sensitivity analysis of cost-effectiveness results for oseltamivir vs. usual care: means (parentheses minimum and maximum) (D dominates in the incremental analysis, UC usual care, Oselt. oseltamivir)

\begin{tabular}{|c|c|c|c|c|}
\hline & \multicolumn{2}{|c|}{ Health care payer perspective } & \multicolumn{2}{|l|}{ Society perspective } \\
\hline & Cost per QALY gained $(\xi)$ & $\begin{array}{l}\text { Cost per day of normal } \\
\text { activity gained }(\mathfrak{(})\end{array}$ & Cost per QALY gained ( $(\mathbf{)})$ & $\begin{array}{l}\text { Cost per day of normal } \\
\text { activity gained }(£)\end{array}$ \\
\hline Base case & $5,600(1,403 ;$ UCD $)$ & $14.36(10.69 ; 17.67)$ & Oselt. D (Oselt. D; 15,619ª) & - \\
\hline Diagnostic certainty $34 \%$ & $11,872(1,881 ;$ UCD) & $32.79(25.55 ; 39.07)$ & Oselt. D (Oselt. D; 3,824a) & - \\
\hline Late starters $25 \%$ & $7,826(1,574 ;$ UC D) & $20.20(14.92 ; 24.59)$ & Oselt. D (Oselt. D; 9,702 ${ }^{\mathrm{a}}$ ) & - \\
\hline $\begin{array}{l}\text { Nls have no effect on } \\
\text { hospitalizations, } \\
\text { complications, mortality, } \\
\text { diagnostic certainty } 34 \%\end{array}$ & $20,717(19,217 ; 22,647)$ & $38.80(36.07 ; 42.76)$ & Oselt. D (Oselt. D; Oselt. D) & $\begin{array}{l}\text { Oselt. D (Oselt. D; Oselt. D) } \\
\text { for all analyses }\end{array}$ \\
\hline $\begin{array}{l}\text { No hospitalizations, } \\
\text { complications, mortality, } \\
\text { diagnostic certainty } 34 \%\end{array}$ & $20,704(20,066 ; 21,318)$ & $38.21(37.03 ; 39.34)$ & Oselt. D (Oselt. D; Oselt. D) & - \\
\hline Undiscounted & $5,069(1,110 ;$ UCD $)$ & $14.36(10.69 ; 17.67)$ & Oselt. D (Oselt. D; 10,466ª) & - \\
\hline Low work loss & N/A & N/A & Oselt. D (Oselt. D; 15,619ª) & - \\
\hline
\end{tabular}

analysis a proportion of $25 \%$ "late starter of treatment" (after $48 \mathrm{~h}$ ) is assumed. For the societal perspective oseltamivir still dominates usual care. For the healthcare payer perspective oseltamivir remains cost-effective even with $47.5 \%$ of patients receiving no benefits $(30 \%$ influenza negative plus $25 \%$ late presenters among the influenza positive patients). In the multiway sensitivity analysis, omitting all effects related to hospitalizations, complications, and mortality and assuming a low diagnostic certainty rate of $34 \%$, oseltamivir is still cost effective from the NHS point of view and dominates usual care from the societal point of view. As expected, discounting only has a small impact on the results as does the lower work loss assumed in sensitivity analysis.

\section{Discussion}

Influenza is an uncomfortable condition that severely affects quality of life for a relatively short period but is uncomplicated and self-limiting in most cases. However, our review of the literature suggests that actual knowledge about influenza and its consequences on health, healthcare resource use, and the economy are limited. As part of our model we tried to capture relevant published knowledge about the disease and its implications. However, in the context of decision making about providing treatment with neuraminidase inhibitors for influenza it is important to understand that the results presented here are conditional on the baseline probabilities used in the model. Our model is subject to a number of limitations. In general, there is a lack of accurate information about influenza complication, mor- 
tality, and hospitalization rates in the otherwise healthy adult population. All baseline probabilities for influenza complications, mortality, and hospitalization are from rather few publications due to the relatively limited knowledge of the true complication and mortality rates associated with influenza. Also worth noting is that the baseline probabilities are based on ILI and not confirmed influenza cases. If it had been possible to filter out ILI cases due to other pathogens (e.g., respiratory syncytial virus, parainfluenzavirus, coronavirus) from true influenza cases, the baseline probabilities would most likely be higher. Despite the availability of rapid influenza tests, testing is not routinely carried out as it is not cost-effective [32]. The baseline probabilities reflect the influenza seasons in which the studies were conducted (all of which were interpandemic seasons). This means that in the case of a particularly strong influenza epidemic, or a pandemic, the baseline incidence rates of complications, mortality and hospitalization would probably be substantially higher, as would be the certainty of diagnosis [33]. Hence the resulting cost-effectiveness of treatment would be even more favorable (assuming the same treatment effect). Due to data limitations we also did not include hospitalizations due to bronchitis, nor did we include certain other secondary complications (e.g., asthmatic exacerbations or cardiac events). However, the exclusion of all of these events from our model is likely to generate conservative estimates of the benefits of influenza treatment.

Our analysis is also limited by the availability of accurate UK medical resource use data, which were not collected alongside clinical trials. Our estimates of resource use are based partly upon non-UK resource data combined with UK-specific unit costs. While some data are available based upon retrospective analysis of patient records in the UK [1], the inclusion of data from NAMCS [10] reflects both the detailed breakdown of resource use by complication and the similarity of aggregate data on resource use to comparable UK sources. On the other hand, the model captures as much of the influenza illness as possible given the published data and uses probabilistic modeling taking into ac- count the uncertainty pertaining to the complication, hospitalization, and mortality rates and durations (days to return to normal activity, length of stay in hospital) that drives the dynamics of influenza and influenza treatment.

Patient preferences were obtained in clinical trials by using rating scales. As opposed to the standard gamble method, the question was framed under certainty, and the subjects were asked to perform a scaling task based on introspection. Therefore the rating scale method does not require the subject to choose between alternatives and does not capture the subject's risk attitude. Research has shown that standard gamble scores are higher than time trade-off scores, which are higher than visual analogue scores [26]. Biases that have been reported related to rating scales are the end-of-scale bias (subjects tend not to use the ends of the scale) and the spacing out bias (subjects tend to space out the outcomes over the scale) [26]. However, the rating scale method is a widely accepted method for measuring patient's preferences as it is quick and efficient. Furthermore, rating scales can be converted to time trade-off scores using power-curve conversion [26].

A day of normal activity gained is useful as a disease-specific outcome that enables us to make comparisons between the interventions under consideration here. However, interpreting the results is not straightforward. We report net costs for a day of normal activity gained with oseltamivir compared to usual care to be $£ 14$ for otherwise healthy adults. In general, $£ 14$ for a day of normal activity in a healthy adult population is likely to represent good value for money, assuming an earlier return to work and an average gross daily wage of approx. $£ 80$ [11].

We present the simulation results using the mean, minimum, and maximum values for cost-effectiveness ratios because of the problems related to expressing stochastic uncertainty surrounding the costeffectiveness ratio. The main problem of which is the discontinuous distribution of the cost-effectiveness ratio. Several methods for estimating confidence intervals for cost-effectiveness ratios have been proposed, each posing methodological challenges. One of the alternative approaches is the use of acceptability curves, which are also presented for our analysis. Assuming that the cost-effectiveness ratio is symmetrical, the $50 \%$ point corresponds to the point estimate of the cost-effectiveness ratio. Furthermore, when the curves increase monotonically, cutting $2.5 \%$ from either end of the vertical axis and using the curve to map these values on to the horizontal axis defines the $95 \%$ confidence interval. However, a limitation to this method is that preferences for gains and losses are in fact not symmetrical, i.e., the 50\% point would not correspond to the mean value of the incremental cost-effectiveness ratio [34].

It is important to consider our results in the context of other published cost-effectiveness analyses of influenza treatment in the UK. Analyses presented with the most recent guidance regarding the use of oseltamivir, zanamivir, and amantadine by NICE [35] report an incremental cost per QALY for oseltamivir of $£ 19$,015/QA-

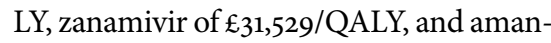
tadine of $£ 11,830 / Q A L Y$ compared to usual care in the base case (assuming $47 \%$ diagnostic certainty and no impact on hospitalizations and mortality) for the otherwise healthy adult population. The sensitivity analysis on alternative model specifications (70\% diagnostic certainty) shows the following incremental cost per QALY compared to usual care: amantadine $£ 5,532$, oseltamivir $£ 11,389$, and zanamivir $£ 20,000$, which are comparable to the results presented in our study.

\section{Conclusion}

Given the present published knowledge about influenza disease and its associated complications, our analysis found that oseltamivir is a cost-effective treatment for influenza compared to usual care and to zanamivir in the otherwise healthy adult population. The incremental cost-effectiveness ratios comparing oseltamivir to usual care are $£ 14-39$ per day of activity gained and are $£ 5,600-20,717 /$ QALY from the health care payer perspective. Probabilistic modeling, used to explore the uncertainty around the base case mean expected cost-effectiveness ratios, demonstrates that the simulated estimates are consistent with a conclusion that oseltamivir is cost- 
effective in the population group evaluated. From a societal perspective treatment with oseltamivir dominates usual care and zanamivir providing health benefits at lower total costs.

\section{Corresponding author \\ Beate Sander}

Institute for Medical Outcome Research $\mathrm{GmbH}$, Lörrach, Germany

e-mail: bsander@uhnres.utoronto.ca

\section{Acknowledgements}

Financial support for this study was provided entirely by a contract with F. Hoffmann-La Roche Ltd., Pharmaceuticals Division. The funding agreement ensured the authors' independence in designing the study, interpreting the data, writing, and publishing the report. M.G. and J.M. are employed by the sponsor.

\section{References}

1. Meier C, Napalkov $P$, Wegmuller $Y$, Jefferson T, Jick $\mathrm{H}$ (2000) Population-based study on incidence, risk factors, clinical complications and drug utilisation associated with influenza in the United Kingdom. Eur J Clin Microbiol Infect Dis 19:834-842

2. National Institute for Clinical Excellence (2003) Guidance on the use of zanamivir, oseltamivir and amantadine for the treatment of influenza. Technology appraisal guidance no 58 . http://www.nice. org.uk/pdf/58_Flu_fullguidance.pdf

3. Baltussen R, Reinders A, Sprenger M, Postma M, Jager J, Ament A et al. (1998) Estimating influenza-related hospitalization in The Netherlands. Epidemiol Infect 121:129-138

4. Simonsen L, Fukuda K, Schonberger L, Cox N (2000) The impact of influenza epidemics on hospitalizations. J Infect Dis 181:831-837

5. Kaiser L, Keene O, Hammond J, Elliott M, Hayden F (2000) Impact of zanamivir on antibiotic use for respiratory events following acute influenza in adolescents and adults. Arch Intern Med 160:3234-3240

6. Bochud P, Moser F, Erard P, Verdon F, Studer J, Villard $G$ et al. (2001) Community-acquired pneumonia. A prospective outpatient study. Medicine (Baltimore) 80:75-87

7. Spitäler der Schweiz (1997) H+ Spitalstatistiken. Medizinische Gesamtstatistik der Schweizer Spitäler. Aarau

8. Anonymous (2001) British national formulary no 42. British Medical Association and Royal Pharmaceutical Society of Great Britain: London

9. Netten A, Rees T, Harrison G (2001) Unit costs of health and social care. In: London School of Economics and University of Manchester (eds) Personal Social Services Research Unit at the University of Kent at Canterbury: Canterbury

10. Anonymous (1997) National Ambulatory Medical Care Survey (NAMCS). In: National Centre for Health Statistics NCHS (ed) Department of Health and Human Services

11. Anonymous (2001) New earnings survey 2000, rev edn. Office for National Statistics: London
12. Nicholson KG, Aoki FY, Osterhaus AD, Trottier S, Carewicz O, Mercier CH et al. (2000) Efficacy and safety of oseltamivir in treatment of acute influenza: a randomised controlled trial. Neuraminidase Inhibitor Flu Treatment Investigator Group. Lancet 355:1845-1850

13. Treanor JJ, Hayden FG, Vrooman PS, Barbarash $R$, Bettis R, Riff D et al. (2000) Efficacy and safety of the oral neuraminidase inhibitor oseltamivir in treating acute influenza: a randomized controlled trial. US Oral Neuraminidase Study Group. JAMA 283:1016-1024

14. Kaiser L, Wat C, Mills T, Mahoney P, Ward P, Hayden $F$ (2003) Impact of oseltamivir treatment on influenza-related lower respiratory tract complications and hospitalizations. Arch Intern Med 163:16671672

15. Vindt HM, Gyldmark $M$, Holme HE, Vindt Holm M, Gyldmark M, Holme Hansen E (2004) Pharmacoeconomic assessment of oseltamivir in treating influenza-the case of otherwise healthy Danish adolescents and adults. Pharm World Sci 26:339345

16. Gyldmark M, Wegmueller Y, Barker C (2002) The impact of influenza on individuals' daily activities, the effect of treatment with oral oseltamivir on return to normal health. World Organisation of Family Doctors (WONCA) conference, London

17. Makela MJ, Pauksens K, Rostila T, Fleming DM, Man CY, Keene ON et al. (2000) Clinical efficacy and safety of the orally inhaled neuraminidase inhibitor zanamivir in the treatment of influenza: a randomized, double-blind, placebo-controlled European study. J Infect 40:42-48

18. Thompson W, Shay D, Weintraub E, Brammer L, Cox N, Anderson Let al. (2003) Mortality associated with influenza and respiratory syncytial virus in the United States. JAMA 8 289:179-186

19. Burls $A(2000)$ Is zanamivir effective for the treatment of influenza in adults? Submission to NICE. West Midlands Development and Evaluation Service

20. Nicholson K, Webster R, Hay A (1998) Textbook of influenza. Blackwell: London

21. Diggory P, Fernandez C, Humphrey A, Jones V, Murphy M (2001) Comparison of elderly people's technique in using two dry powder inhalers to deliver zanamivir: randomised controlled trial. BMJ 322:577-579

22. Stouthard M, Essink-Bot M, Bonsel G, Barendregt J, Kramer P, van de Water H (1997) Disability weights for diseases in The Netherlands. Department of Public Health, Erasmus University Rotterdam: Rotterdam

23. Turner D, Wailoo A, Nicholson $K$, Cooper N J, Sutton AJ, Abrams KR (2002) Systematic review and economic decision modelling for the prevention and treatment of influenza A and B. NICE assessment report

24. Briggs A (2000) Handling uncertainty in cost-effectiveness models. Pharmacoeconomics 17:479-500

25. Hunink M, Glasziou P, Siegel J, Weeks J, Pliskin J, Elstein A et al. (2001) Decision making in health and medicine. Integrating evidence and values. Cambridge University Press: Cambridge

26. Drummond M, O'Brien B, Stoddart G, Torrance G (1997) Methods for the economic evaluation of health care programmes, 2nd edn. Oxford University Press: Oxford

27. Kielhorn A, Graf von der Schulenburg J (2000) The health economics handbook, 2nd edn. Adis International Limited: Chester
28. Sculpher M (2001) The role and estimation of productivity costs in economic evaluation. In: Drummond $\mathrm{M}$ (ed) Economic evaluation in health care: merging theory with practice. pp 94-112

29. National Institute for Clinical Excellence (2001) Guidance for manufacturers, sponsors. Technological appraisals process series 5 . London

30. European Agency for the Evaluation of Medicinal Products (2002) European public assessment report (EPAR): summary of product characteristics. http://www.eudra.org/humandocs/Humans/EPAR/ tamiflu/tamiflu.htm

31. Akazawa M, Sindelar J, Paltiel A (2003) Economic costs of influenza-related work absenteeism. Value Health 6:107-115

32. Schwarzinger $M$, Housset $B$, Carrat $F$ (2003) Bedside rapid flu test and zanamivir prescription in healthy working adults: a cost-benefit analysis. Pharmacoeconomics 21:215-224

33. Meltzer Ml, Cox N, Fukuda K (1999) The economic impact of pandemic influenza in the United States: priorities for intervention. Emerg Inf Dis 5:659-671

34. Glick HA, Briggs HA, Polsky D (2001) Quantifying stochastic uncertainty and presenting results of cost-effectivenss analyses. Expert Rev Pharmacoecon Outcomes Res 1:89-100

35. National Institute for Clinical Excellence (2000) Guidance on the use of zanamivir (relenza) in the treatment of influenza. Document no 15. NICE: London 